\title{
THE
}

\section{Motivational interviewing to reduce substance-related consequences: Effects for incarcerated adolescents with depressed mood}

\author{
L.A.R. Stein \\ University of Rhode Island, larstein@uri.edu \\ Mary Clair \\ University of Rhode Island \\ Rebecca Lebeau \\ University of Rhode Island \\ Suzanne M. Colby \\ Nancy P. Barnett \\ Follow this and additional works at: https://digitalcommons.uri.edu/psy_facpubs \\ This is a pre-publication author manuscript of the final, published article. \\ See next page for additional authors \\ Creative Commons License \\ (c) (i) (8)
}

This work is licensed under a Creative Commons Attribution-Noncommercial-No Derivative Works 4.0 License.

\section{Citation/Publisher Attribution}

L.A.R. Stein, et al. "Motivational interviewing to reduce substance-related consequences: Effects for incarcerated adolescents with depressed mood." Drug and Alcohol Dependence, vol. 118, no. 2-3, 2011, pp. 475-478. doi: 10.1016/j.drugalcdep.2011.03.023

Available at: https://doi.org/10.1016/j.drugalcdep.2011.03.023

This Article is brought to you for free and open access by the Psychology at DigitalCommons@URI. It has been accepted for inclusion in Psychology Faculty Publications by an authorized administrator of DigitalCommons@URI. For more information, please contact digitalcommons-group@uri.edu. 


\section{Authors}

L.A.R. Stein, Mary Clair, Rebecca Lebeau, Suzanne M. Colby, Nancy P. Barnett, Charles Golembeske, and Peter M. Monti 


\title{
Motivational Interviewing to Reduce Substance-Related Consequences: Effects for Incarcerated Adolescents with Depressed Mood
}

\author{
L.A.R. Stein a,b,c, Mary Clair ${ }^{\mathrm{a}}$, Rebecca Lebeaua ${ }^{\mathrm{a}}$, Suzanne M. Colby ${ }^{\mathrm{c}}$, Nancy P. Barnett ${ }^{\mathrm{c}}$, \\ Charles Golembeske ${ }^{b}$, and Peter M. Monti ${ }^{c}$ \\ a Social Sciences Research Center, University of Rhode Island, 2 Chafee Road, Kingston, RI \\ 02881 \\ b The Rhode Island Training School, 300 New London Avenue, Cranston, RI 02920 \\ c Center for Alcohol and Addiction Studies, Brown University 121 South Main Street, Providence, \\ RI 02906
}

\section{Abstract}

Background-The impact of depressed mood on Motivational Interviewing (MI) to reduce risky behaviors and consequences in incarcerated adolescents was examined in this brief report.

Methods-Adolescents $(\underline{N}=189)$ were randomly assigned to receive MI or Relaxation Training (RT).

Results-At 3-month follow-up assessment, MI significantly reduced risks associated with marijuana use, with a trend towards reducing risks associated with alcohol use. There was also a trend for depressive symptoms to be associated with reduced risks after release. Interaction effects were non-significant, indicating no moderating effects for depressed mood on treatment outcome.

Conclusions-MI may be a useful treatment for incarcerated adolescents in order to reduce risks and consequences associated with substance use after release.

\section{Keywords}

Adolescents; incarceration; risky behavior; treatment; dysphoria

\section{Introduction}

Juvenile detainees have high rates of alcohol and marijuana use disorders, as well as depressive disorders (Teplin et al., 2002). Similarly the prevalence of comorbid affective and substance use disorders among them is high (Abram et al., 2003). In addition, incarcerated adolescents with negative mood endorse more consequences from alcohol and marijuana use than their normal counterparts (Tuner et al., 2005).

\footnotetext{
(c) 2011 Elsevier Ireland Ltd. All rights reserved.

Please address all correspondence to: L. A. R. Stein, Social Sciences Research Center, University of Rhode Island, 2 Chafee Rd., Kingston, RI 02881, 401-874-4261 (phone); Fax: 401-874-5562, LARStein@URI.EDU.

Publisher's Disclaimer: This is a PDF file of an unedited manuscript that has been accepted for publication. As a service to our customers we are providing this early version of the manuscript. The manuscript will undergo copyediting, typesetting, and review of the resulting proof before it is published in its final citable form. Please note that during the production process errors may be discovered which could affect the content, and all legal disclaimers that apply to the journal pertain.
} 
Few studies have investigated substance use interventions with incarcerated adolescents; however, Motivational Interviewing (MI) has shown promising results. MI may be particularly well-suited to adolescents, given their sensitivity and resistance to adult attempts to control or direct their behavior (Marlatt and Witkiewitz, 2002; Masterman and Kelly, 2003). One randomized trial found that substance-involved incarcerated adolescents that received MI as compared to Relaxation Training (RT) engaged more in substance use treatment 2 months into standard facility care (Stein et al., 2006a). Further, adolescents low in depressive symptoms that received MI had lower rates of risky sexual behaviors (Rosengard et al., 2007) and driving under the influence (Stein et al., 2006b) as compared to those that received RT. A recent related study from this trial found that at 3 month followup, adolescents who received MI had lower rates of alcohol and marijuana use as compared to those who received RT (Stein et al., 2010b).

Depression among substance users is prevalent (Daughters et al., 2008), but the impact of depression on outcomes has yielded inconsistent findings (Kranzler et al., 1996). Depressive symptoms at entry into substance treatment have been associated with attrition (Curran et al., 2002) in adults, and more depressive symptoms early in residential substance treatment have related to poorer substance outcomes for adolescents (Subramaniam et al., 2007). On the other hand, adults with comorbid major depression early in inpatient alcohol treatment evidenced less drinking at follow-up than those without depression (Kranzler et al., 1995); and similarly, adults showing significant depression early in addictions treatment had longer duration of abstinence at follow-up (Charney et al., 1998). Tapert and colleagues (2003) suggested that depressed mood might make adolescents more amenable to brief intervention for substance use, or, conversely, that for some adolescents it may be a treatment liability (perhaps by impeding the ability to attend to intervention or mobilize resources). The current brief report investigated the impact of depressed mood on MI to reduce risky behaviors and consequences associated with alcohol and marijuana use in incarcerated adolescents. Previous work cited above (Rosengard et al., 2007; Stein et al., 2006b) focused on frequency of specific substance-related risk-taking behaviors (condom use, risky driving) part way through the trial, whereas the present study examines both risks and consequences associated with substance use as measured by scales at the conclusion of the trial.

\section{Methods}

\subsection{Participants}

The study was conducted in compliance with Brown University's Institutional Review Board. Participants were recruited from a state juvenile correctional facility in the Northeast (April, 2001 to March, 2006). Adolescents were identified as potential participants for the study immediately after adjudication if they were $14-19$ years and were sentenced to the facility for 4-12 months. Adolescents provided assent and written consent was obtained from legal guardians (adolescents $\geq 18$ years provided consent). Guardians and adolescents were informed that all information was confidential except reports of child abuse/neglect, plans to escape, or threats to hurt self or others. Adolescents were eligible if they engaged in at least monthly marijuana use or binge-drinking in the year before incarceration, or if they used any alcohol or marijuana in the month prior to incarceration (or prior to the offense leading to incarceration).

One hundred eighty nine participants completed baseline assessment (about 5 weeks after facility entry). At 3-month post-release follow-up, 181 were re-interviewed; five could not be located for follow-up, and three adolescents withdrew from the study prior to completion of the 3-month follow-up. The sample $(\underline{N}=189)$ was comprised of the following racial/ ethnic backgrounds: $32.8 \%$ white, $29.1 \%$ Hispanic, $28.0 \%$ African American, 3.7\% NativeAmerican, 3.2\% Asian-American, and 3.2\% self-identified as "other." A large percentage 
were boys $(85.7 \%)$, with an average age $(\underline{\mathrm{M}})$ of 17.12 years (standard deviation, $\underline{\mathrm{SD}}=1.10$ ). Sixty-three percent and $88.9 \%$ qualified for alcohol and marijuana use disorders, respectively, in the previous year according to diagnostic interview.

\subsection{Interventions}

In preparation for the facility's standard care substance use treatment, participants were randomly assigned to and received intervention (MI or RT) shortly after the baseline assessment. Randomization was accomplished via random numbers table in advance and placed in a sealed envelope by the Project Coordinator. Interventions were about 90 minutes at baseline and about 60 minutes at booster (provided within 2 weeks of release to address variation in time incarcerated), and each research counselor conducted both interventions. Treatments were manualized, and intensive training was provided as was weekly supervision to maintain a high degree of fidelity (see O'Leary-Tevyaw and Monti, 2004; Stein et al., 2006a). The MI protocol was based on the principles of MI (Miller and Rollnick, 2002), with focus on empathy, not arguing, developing discrepancy, self-efficacy, and personal choice. Reducing alcohol and/or marijuana use and associated risky behaviors and consequences of use were a focus of the intervention. The RT protocol was delivered by research counselors to control for the effects of receiving an individual intervention. RT included progressive muscle relaxation, use of guided imagery, feedback on use of techniques, and general advice to stop risky behaviors related to alcohol and marijuana use.

\subsection{Measures}

The Risks and Consequences Questionnaire (RCQ) measures problems associated with alcohol and marijuana use (missing school, relationship difficulty, etc.). At baseline it covers 12 months pre-incarceration and at 3 months after release it covers 90 days postincarceration. Alcohol (RCQ-A) and marijuana (RCQ-M) scales (11 items, each) are scored according to whether events occurred (yes/no). It is reliable and valid for use with incarcerated adolescents, with Cronbach alpha ranging from .72 - .83 (Stein et al., 2010a). The Center for Epidemiological Studies-Depression scale (CES-D) was administered at baseline. It is reliable and valid for use with adolescents; scores of $\geq 16$ indicate presence of significant depressive symptomatology during the past week (Radloff, 1977); and 68.5\% of the sample met this cut-off.

Trained Research Assistants (RAs) conducted follow-up assessment and were blind to intervention condition. More detailed description of the methodology used in this study can be found in Stein et al., (2006a) and Stein et al., (2006b).

\subsection{Analyses}

Analysis of covariance (ANCOVA) was performed to determine the impact of depressed mood on treatment to reduce risky behaviors and consequences. Dependent variables (DVs) at 3-month post-release assessment were RCQ-A and RCQ-M. For each ANCOVA, the covariate was the corresponding baseline measure of the DV, and the independent variables (IVs) were intervention condition and depressive symptoms. Outcome analyses for main effects and interactions were powered at 0.90 for $\underline{\alpha}$ set at $.05, \underline{N}=181$, and effect sizes in the medium range (Borenstein et al., 2000; Cohen, 1988).

\section{Results}

No significant differences were found between treatment groups on baseline variables including gender, age, ethnicity/race, depressive symptoms, participation in facility substance use treatment, or mother's education level, nor did groups differ on key variables such as length of incarceration or use of treatment after release. Means and standard 
deviations for covariates at baseline are in Table 1. Main effects and interactions are in Table 2. A significant main effect $(\mathrm{p} \leq .007)$ was found for treatment group on risks and consequences for marijuana (RCQ-M), with participants in the MI intervention showing fewer problems on the RCQ-M than participants in the RT condition. A marginal effect ( $\mathrm{p}$ $\leq .096$ ) was found for treatment on risks and consequences for alcohol (RCQ-A).

Participants who received MI indicated fewer problems on the RCQ-A, as compared to RT participants. Additionally, a main effect for depressive symptoms approached significance $(\mathrm{p} \leq .051)$, such that the high depressive symptom group reported fewer risky behaviors and consequences on the RCQ-A as compared to the low depressive symptom group. No main effect of depressive symptoms was found on the RCQ-M, and no significant interaction effects were found.

\section{Discussion}

Findings of this brief report indicate MI delivered to incarcerated adolescents significantly impacted subsequent risks and consequences associated with marijuana use in incarcerated adolescents with a trend towards impacting problems associated with alcohol use. Given the high prevalence of marijuana and alcohol disorders in this population $(88.9 \%$ and $63 \%$ respectively, in the past year), these results show promise for MI decreasing difficulties associated with marijuana and alcohol use in incarcerated adolescents. There was also a trend for depressive symptoms to be associated with reduced problems associated with alcohol use after release. Treatment by depressive symptom interaction effects were nonsignificant, indicating that depressive symptoms early in incarceration do not impact treatment effects.

In a population that frequently uses alcohol and marijuana, identifying a brief intervention that reduces risky behaviors related to these drugs is of much significance. Although there was a trend for higher baseline depressive symptoms to be related to fewer alcohol-related difficulties at follow-up (perhaps because adolescents must exert more effort to access alcohol than marijuana), it is important to further evaluate and treat adolescents with depressive symptoms early in incarceration. Of note, Stein et al. (2010b) found more pronounced effects for MI on alcohol use as compared to marijuana use. The current study suggests MI may impact marijuana-related behaviors in terms of harm reduction (reducing harmful behaviors related to marijuana use).

Limitations of this study include reliance on self-report methods, a brief follow-up period, lack of a no-treatment control group, and the fact that depressive symptoms were assessed during the past week only. However, methods of self-report are generally accurate (Babor et al., 2002), brief follow-ups are often used to first establish an effect for treatment, and use of a no-treatment group would not have allowed us to control for attention effects. Our measure of depressive symptoms may reflect current stressors and long-standing dysphoria, thus future studies may assess lifetime and current major depressive disorder. Replication with larger samples, including more females, and longer follow-ups to determine if treatment effects are maintained should be targets for future research.

Although prior work suggested that the effects of MI might be influenced by depressive symptoms, this study did not find evidence for the moderating influence of depressive symptoms. The current study indicates that MI is a viable treatment for incarcerated adolescents in order to reduce substance-related risks and consequences, and that depressive symptoms early in incarceration do not impact treatment effects. 


\section{References}

Abram KM, Teplin LA, McClelland GM, Dulcan MK. Comorbid psychiatric disorders in youth in juvenile detention. Arch Gen Psychiatry. 2003; 60:1097-1108. [PubMed: 14609885]

Babor TF, Webb C, Burleson JA, Kaminer Y. Subtypes of classifying adolescents with marijuana use disorders: construct validity and clinical implications. Addiction. 2002; 97:58-69. [PubMed: 12460129]

Borenstein, M.; Rothstein, H.; Cohen, J. Power and Precision. Release 2.0. NIMH and Biostaistics; Bethesda, MD: 2000.

Charney DA, Paraherakis AM, Negrete JC, Gill KJ. The impact of depression on the outcome of addictions treatment. J Subst Abuse Treat. 1998; 15:123-130. [PubMed: 9561951]

Cohen, J. Statistical Power Analysis for the Behavioral Sciences. 2. Lawrence Erlbaum Associates; Hillsdale: 1988.

Curran GM, Kirchner JE, Worley M, Rookey C, Booth BM. Depressive symptomatology and early attrition from intensive outpatient substance use treatment. J Behav Health Serv Res. 2002; 29:138 143. [PubMed: 12032971]

Daughters SB, Braun AR, Sargeant MN, Reynolds EK, Hopko DR, Blanco C, Lejuez CW. Effectiveness of a brief behavioral treatment for inner-city illicit drug users with elevated depressive symptoms: the Life Enhancement Treatment for Substance Use (LETS Act!). J Clin Psychiatry. 2008; 69:122-129. [PubMed: 18312046]

Kranzler HR, Del Boca RK, Rounsaville BJ. Comorbid psychiatric diagnosis predicts three-year outcomes in alcoholics: a post-treatment natural history study. J Stud Alcohol. 1996; 57:619-626. [PubMed: 8913993]

Marlatt GA, Witkiewitz K. Harm reduction approaches to alcohol use: health promotion, prevention, and treatment. Addict Behav. 2002; 27:867-886. [PubMed: 12369473]

Masterman PW, Kelly AB. Reaching adolescents who drink harmfully: fitting intervention to developmental reality. J Subst Abuse Treat. 2003; 24:347-355. [PubMed: 12867209]

Miller, WR.; Rollnick, S. Motivational Interviewing: Preparing People for Change. 2. Guilford Press; New York: 2002.

O'Leary-Tevyaw T, Monti PM. Motivational enhancement and other brief interventions for adolescent substance abuse: foundations, applications, and evaluations. Addiction. 2004; 99 (Suppl 2):63-75. [PubMed: 15488106]

Radloff LS. The use of the Center for Epidemiologic Studies Depression Scale in adolescents and young adults. J Youth Adolesc. 1991; 20:149-166.

Radloff LS. CES-D scale: a self-report depression scale for research on the general population. Appl Psychol Meas. 1977; 1:385-401.

Rosengard C, Stein LAR, Barnett NP, Monti PM, Golembeske C, Lebeau-Craven R, Miranda R. Randomized clinical trial of motivational enhancement of substance use treatment among incarcerated adolescents: post-release condom non-use. J HIV AIDS Prev Child Youth. 2007; 8:45-64. [PubMed: 19809580]

Stein LAR, Colby SM, Barnett NP, Monti PM, Lebeau-Craven R, Miranda R. Enhancing substance abuse treatment engagement in incarcerated adolescents. Psychol Serv. 2006a; 3:25-34. [PubMed: 20617117]

Stein LAR, Colby SM, Barnett NP, Monti PM, Golembeske C, Lebeau-Craven R. Effects of motivational interviewing for incarcerated adolescents on driving under the influence after release. Am J Addict. 2006b; 15:50-57. [PubMed: 17182420]

Stein LAR, Lebeau R, Clair M, Rossi JS, Martin RM, Golembeske C. Validation of a measure to assess alcohol- and marijuana-related risks and consequences among incarcerated adolescents. Drug Alcohol Depend. 2010a; 109:104-113. [PubMed: 20149558]

Stein, LAR.; Lebeau, R.; Colby, SM.; Barnett, N.; Golembeske, C.; Monti, P. Effects of Motivational Interviewing for incarcerated adolescents on reducing alcohol and marijuana use after release. University of Rhode Island; 2010b. Manuscript submitted for publication 
Subramaniam GA, Stitzer MA, Clemmey P, Kolodner K, Fishman MJ. Baseline depressive symptoms predict poor substance use outcome following adolescent residential treatment. J Am Acad Child Adolesc Psychiatry. 2007; 46:1062-1069. [PubMed: 17667484]

Tapert SF, Colby SM, Barnett NP, Spirito A, Rohsenow DJ, Myers MG, Monti PM. Depressed mood, gender, and problem drinking in youth. J Child Adolesc Subst Abuse. 2003; 12:55-68.

Teplin LA, Abram KM, McClelland GM, Dulcan MD, Mericle AA. Psychiatric disorders in youth in juvenile detention. Arch Gen Psychiatry. 2002; 59:1133-1143. [PubMed: 12470130]

Turner AP, Larimer ME, Sarason IG, Trupin EW. Identifying a negative mood subtype in incarcerated adolescents: relationship to substance use. Addict Behav. 2005; 30:1442-1448. [PubMed: 16022939] 


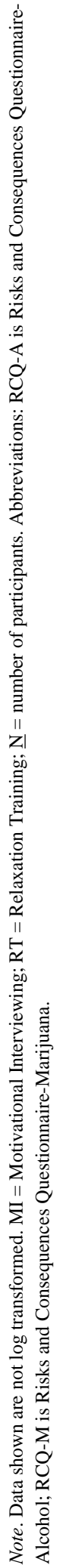

Drug Alcohol Depend. Author manuscript; available in PMC 2012 November 1. 
